\title{
Functional usability analysis of top korean mobile role playing games based on user interface design
}

\author{
Seung Hoon Lee ${ }^{1}$, Doo Heon Song ${ }^{2}$ \\ ${ }^{1}$ Department of Virtual Reality Contents, Young San University, Busan 48015, Korea \\ ${ }^{2}$ Department of Computer Games, Yong-In SongDam College, Yong-in 17145, Korea
}

\section{Article Info \\ Article history: \\ Received Jul 13, 2018 \\ Revised Oct 8, 2018 \\ Accepted Oct 25, 2018}

\section{Keywords:}

Heuristics

Mobile Platform

Role Playing Game

Usability

User interface

\section{Corresponding Author:}

Doo Heon Song,

Department of Computer Games,

Yong-In SongDam College,

571-1 Cheo-in Gu, Yong-in 17145, Korea.

Email : dsong@ysc.ac.kr

\begin{abstract}
Mobile Tole Playing Games (RPGs) are now very popular in gaming market. Still, we have great constraint in hardware provided such as screen size and interaction speed. Adopting techniques such as auto play enables the game designers to weaken the burdens of porting RPG scales to mobile platform. However, the interface design in mobile platform becomes quite different from PC based platform. In this paper, we investigate how user interface functionalities are provided for popular mobile RPGs based on google play downloaded between the year of 2014 and 2016. We analyze the commonalities and the differences of recent successful mobile RPGs first and take a user survey with heuristics. In result, subjects who are $16 \sim 25$ in age weakly accept current interface layout and control as over $70 \%$ positive rate in 4 point Likert scale test.
\end{abstract}

Copyright () 2018 Institute of Advanced Engineering and Science. All rights reserved.

\section{INTRODUCTION}

In game interface design, the rule of thumb is to give good, convenient control over objects provided by the game with enough information and easy access. It can be summarized as the functionality and the usability of the user interface [1]. For role playing games (RPG), which needs the most complex control over objects and feedback of the game, it is quite essential to give proper interface that typically are composed of status window, chatting window, navigation, shortcut keys, and inventory section [2]. Thus, the interface should have the layout containing as rich information as possible but not give overwhelming information to users for not hindering the immersion [3].

However, for mobile RPG, the design principle should be quite different from that of PC based RPG due to the screen size and other hardware constraints. It is important to control the scene changes with respect to the information provided [2].

The usability evaluation issue is more complex. Usability evaluation based on a set of heuristics provided by experts and validated by user surveys becomes more important than ever. However, it also draws plenty of discussions and different approaches on how much the set of heuristics should include user experience, flow and fun issues in conjunction with the functionality [4-10]. Furthermore, it is also found that different genre requires different points in satisfaction of the user interface design such that the casual game [11] and management simulation game [12] users may need different standard of satisfaction.

Thus, in this paper, we will focus on mobile RPG interface. By doing so, we can avoid endless debates on the range of covering aspects of the game contents in usability evaluation $[5,7,8,10]$. First, we will analyze top 5 games of the year between 2014 and 2016 based on google play downloads in Korea [13]. In this analysis, we try to find the commonalities and the differences of the top games and the recent trends 
changing if any exists. Then, we set up a set of heuristics to evaluate solely the satisfaction rate of user interface based on the traditional heuristics by Korhonen et. als [4, 14] and tested by Korean gamers in validity [3] with interpretation of RPG scope. Thus, the evaluation of usability in this paper should be interpreted as the functionality satisfaction since RPG genre contains simply too many factors other than user interface design.

\section{ANALYSIS OF TARGET MOBILE RPG GAMES}

\subsection{USER INTERFACE}

Table 1 summarizes top 5 downloaded mobile RPG between the year of 2014 and 2016 based on [8]. Those games become the set of games we analyze in this paper.

Table 1. Top 5 Mobile RPG between 2014 and 2016

\begin{tabular}{ccc}
\hline 2014 & 2015 & 2016 \\
\hline Hero for Kakao & HIT & Lineage2: Revolution \\
Dark Avengers 2 & Raven & Lineage: Red Knights \\
Crusader Quest & Idea & Destiny Child \\
Seven Knights & Mue origin & Aden \\
Fox Rain & 100HEROES & Summoners War \\
\hline
\end{tabular}

We investigate all above 15 games for the basic layout, battlefield layout, and other functionalities. Figure 1 and Figure 2 demonstrates the difference in basic layout of information objects and battlefield layout with hand control design.

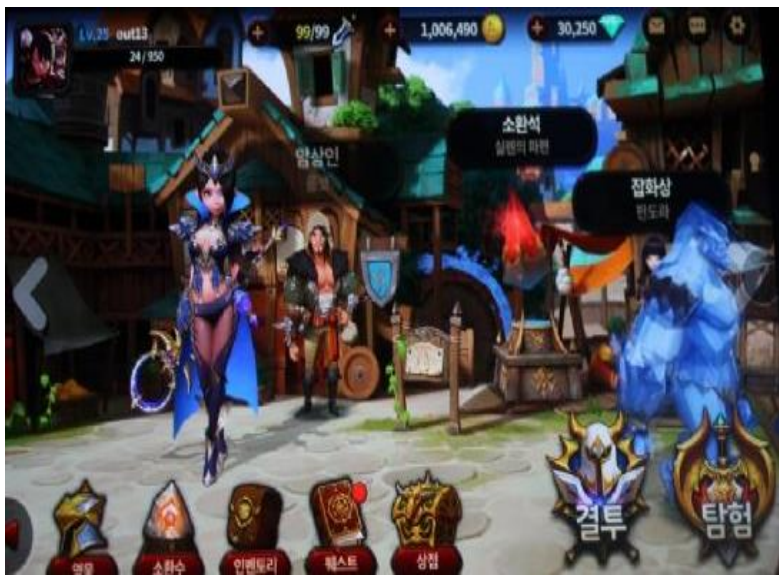

(a) Note Control
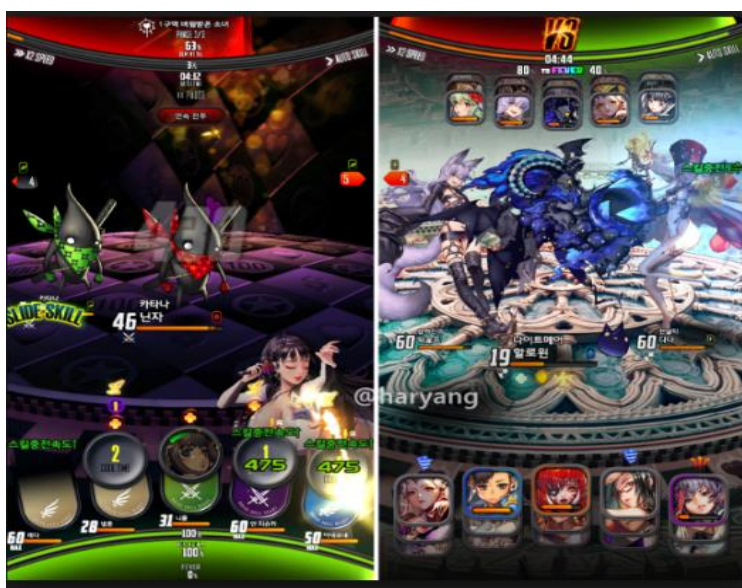

(b) Destiny Child

Figure 1. Basic Interface Examples

The analysis is done by our students having played target games in high levels and at least two months' of experiences by checklist method. Our checklist includes;

a. Chatting window: Can user choose where to put?

b. Main layout: The location on the screen

c. Navigation: Provided?

d. Shortcut Keys: Provided?

e. Inventory Section: On the screen or separated screen?

f. Auto play; Supported?

g. Hand Control: Single hand or both hands?

h. Battle Skill Buttons: The location on the screen 


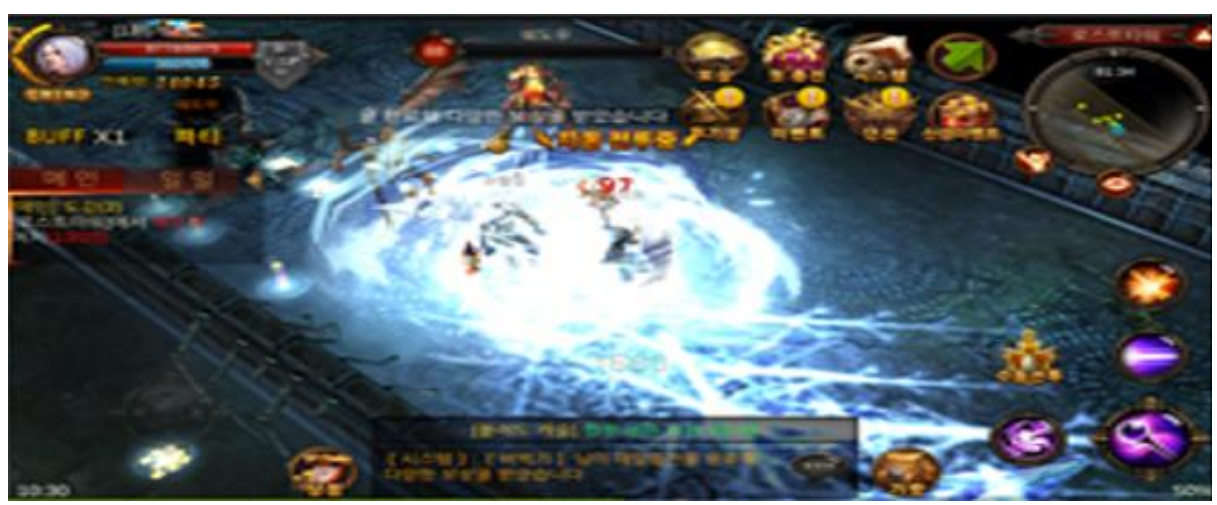

(a) Mue Origine - Controlled by both Hands

(b)

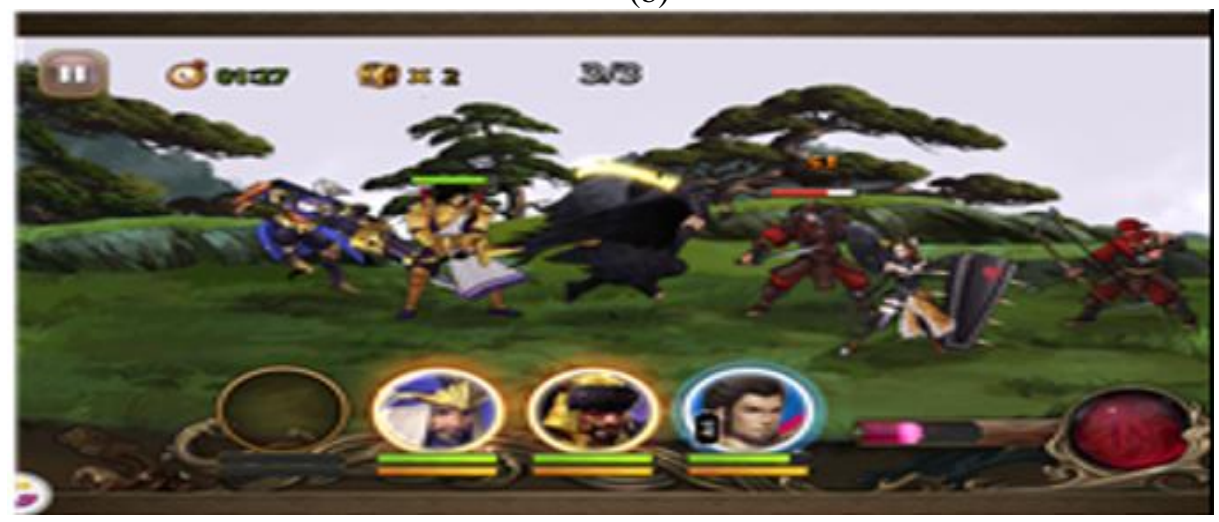

(b) 100 Heroes - Controlled by single Hand

Figure 2. Battlefield Interface and Control Examples

\subsection{Analysis of User Interface}

Table 2, 3, and 4 summarizes the differences found from our analysis in years respectively.

Table 2. Comparative Analysis of 2014 Top 5 RPG

\begin{tabular}{ccccc}
\hline 2014 Games & Chatting Window & Main Layout & Autoplay & Control \\
\hline Hero for Kakao & Choice & Low & Support & Both Hands \\
Dark Avengers 2 & Choice & Upperleft & Support & Both Hands \\
Crusader Quest & Total & Low & No & Single \\
Seven Knights & Choice & Low & Support & Single \\
Fox Rain & Choice & UpperRight & Support & Both Hands \\
\hline
\end{tabular}

Table 3. Comparative Analysis of 2015 Top 5 RPG

\begin{tabular}{ccccc}
\hline 2015 Games & Chatting Window & Main Layout & Autoplay & Control \\
\hline HIT & Choice & Low & Support & Both Hands \\
Raven & Choice & Low & Support & Both Hands \\
Idea & Choice & Low & Support & Both Hands \\
Mue origin & Total & LowerRight & Support & Both Hands \\
100HEROES & Choice & LowerRight & No & Single \\
\hline
\end{tabular}

Table 4. Comparative Analysis of 2016 Top 5 RPG

\begin{tabular}{ccccc}
\hline 2016 Games & Chatting Window & Main Layout & Autoplay & Control \\
\hline Lineage2: Revolution & Total & UpperRight & Support & Both Hands \\
Lineage: Red Knights & Choice & Low & Support & Single \\
Destiny Child & Choice & Low & Support & Single \\
Aden & Choice & Left & Support & Both Hands \\
Summoners War & Choice & LowerRight & Support & Single \\
\hline
\end{tabular}

Functional usability analysis of top korean mobile role playing games based on user... (Seung Hoon Lee) 
Among 15 RPGs we investigated, we found that all of them do not provide shortcut keys nor navigation and inventory section is separated from the battle screen. Also, all investigated games provide battle skill buttons on the lower right part of the battle screen and joystick pad is located on the lower left part. In layout, the top-down layout form was generally applied to the games we analyzed. However, in the case of 'Fox Rain', 'Mu Origin' and 'Lineage Revolution', it is flexible by using the expanded menu format in ' $\neg$ ' shaped layout. In the case of inventory indicator, the important information directly related to the state of the character and affects the battle process, it is provided as a separate menu on the mobile due to the restriction of the screen space as pointed by [2] earlier.

Other than those mentioned differences, most target games have similar user interfaces and controls. Overall, mobile RPGs provide very similar user interfaces thus the next step should be if this typical interface is satisfactory from users' viewpoint. Then, the question will be;

Q: Are gamers comfortable with this user interface and functionality design?

\section{FUNCTIONAL USERBILITY EVALUATION OF YSER INTERFACE There haBY SURVEY}

Heuristic evaluation is one of the so-called expert-based usability inspection methods for software development [15]. Nevertheless, heuristic evaluation with traditional 'usability' heuristics cannot be directly applied to the evaluation of video games because application software and video games are very different in context and usability heuristics do not cover all aspects of gaming such as fun, entertainment, and enjoyment[8].

If it were the userbility evaluation of mobile RPG itself, we may have to consider many aspects other than the functionality of the user interface. As summarized in literature reviews [7,10], one may have to worry if the usability questionnare should include solely on the narrow range of the functionality[5] or include game play/game story, virtual interface and device- and application-specific properties of a system [8]. If that is the latter case, we need a bulky set of heuristics but still, that set of heuristics can never properly represent the complex structure of current mobile RPG due to its intrinsic subjectivity while we want to be as objective as possible in evaluation.

Thus, in this paper, we make our set of questionnare only based on the functionality of user interface. Also, while our questionnare is based on the previous researches [3,4,14], since all target games analyzed were RPG and due to different culture and language, we reinterpretate related heuristics within the scope of RPG genre and make the question as concrete as possible as shown in Table 6.

The survey was done in September 2017 using Google docs and subjects are from many different mobile RPG communities. Total 30 subjects were responded whose age vary between late teens and mid 20's and there were 21 males and 9 females. Their current most played mobile RPG is shown as Table 5.

Table 5. Mobile RPG that Subjects play the most

\begin{tabular}{cc}
\hline Games & Responses \\
\hline Lineage2 Revolution & 7 \\
Crusader Quest & 7 \\
Seven Knights & 7 \\
Destiny Child & 3 \\
Raven & 3 \\
Mue Origin & 1 \\
Idea & 1 \\
HIT & 1 \\
Total & 30 \\
\hline
\end{tabular}

In this survey, we took 4 point scale in order to draw more active opinions from subjects because when we used 5 point scale, non-neglisable portion of responses were just neutral (point 3 ). In our 4 point Liekert scale questionnare, 4 means most satisfactory and 1 denotes the least satisfactory. The result is summarized as shown in Table 6.

The positive responses (response 3 or 4 ) are over $70 \%$ in all questionnare and the absolute points by Liekert scale is between 2.90 and 3.37. The average of total 9 questionnare in Liekert scale is 3.13. Subjects were most satisfactory in finger movement $(\mathrm{Q} 2)$ and clearly visible game indicators in the layout (Q7) but least sarisfactoey on enemy targetting (Q6) and purchasing item convennience (Q1).

On enemy targetting, actually the responses have great discrepancy with respect to the games they played. Destiny child users were most satisfied (all 3 subjects marked as 4) but Seven Knights users gave only 2.71 in average. 
However, as mentioned earlier, subjects were mostly neutral when we used 5 point scale. That means we should not interprete the survey result as indicated in numbers. Rather, gamers may not find better iterfaces in functionality yet and they are not rejecting current interface until then.

Table 6. Functional Userbility Evaluation Result (4 points Liekert scale)

\begin{tabular}{|c|c|c|c|c|c|c|}
\hline Questionnaire/Points & 4 & 3 & 2 & 1 & Positive & Avg. \\
\hline Is purchasing items convenient from UI? & 7 & 14 & 8 & 1 & $70.0 \%$ & 2.90 \\
\hline Are finger movements convenient to use skills? & 16 & 11 & 1 & 2 & $90.0 \%$ & 3.37 \\
\hline Sense of hit satisfactory? & 8 & 15 & 4 & 3 & $76.7 \%$ & 2.93 \\
\hline Are playing screen and map separated clearly? & 11 & 14 & 3 & 1 & $86.2 \%$ & 3.21 \\
\hline Are character movements convenient? & 12 & 11 & 5 & 2 & $76.7 \%$ & 3.10 \\
\hline Is enemy targeting convenient? & 11 & 10 & 5 & 4 & $70.0 \%$ & 2.93 \\
\hline Are indicators clearly visible? & 9 & 18 & 1 & 2 & $90.0 \%$ & 3.13 \\
\hline Are Audio-visual representation and layout satisfactory? & 10 & 16 & 2 & 2 & $86.7 \%$ & 3.37 \\
\hline Is character window convenient to use? & 17 & 10 & 1 & 2 & $90.0 \%$ & 3.13 \\
\hline
\end{tabular}

\section{CONCLUSION}

In this paper, we analyzed the user interface configuration and the battle window display patterns of the fifteen(15) mobile RPGs that were successful in Korean market during 2014 and 2016 (Top 5 in each year). Since RPG is the most dominant game genre among Korean young gamers, either online or mobile platform [16], the usability evaluation of its interface design from actual gamers is important. Thus, we peform a user survey with a set of 9 questionnare based on the heuristic for testing the functionaluty of the user interface and control.

However, due to the nature of the mobile device constraints, the configuration of the interface within the same RPG had little discrepancy in user interface as noted in other genre games (puzzle game [11], management simulation game [12]). In functional userbility test, subjects were positive in general (over $70 \%$ positivelt responded with 3.13 out of 4 point Likert scale) but relatively dissatisfied for the enemy targetting control and item purchasing schemes during game playing. Also, subjects tend to respond as neutral if we gave 5 point scale test. That means even their positive reponses in the survey result should be interpreted as weak acceptance from young gamers.

\section{REFERENCES}

[1] Saunders, K, Novak, J. Game development essentials: Game interface design (2nd Ed.). Boston; Delmar Cengage Learning. 2012.

[2] Lee, JE, Kim, HS. A Study on Scene Changes and Space in UI of Mobile RPG Games, Design Convergence Study. 2017; 16(1). 1-14.

[3] Song, SK, Kim, SH, Lee, J. H. Usability Evaluation of Massively Multi-Player Online Game Design and Key Design Factors, Journal of Korean Design Science. 2006;19(2). 195-206.

[4] Korhonen, H, Koivisto, E. Playability heuristics for mobile games. In Proceedings of the 8th conference on Human-computer interaction with mobile devices and services. ACM. 2006; 9-16.

[5] Pinelle, D, Wong, N, Stach, T. Heuristic evaluation for games: usability principles for video game design. In Proceedings of the SIGCHI Conference on Human Factors in Computing Systems ACM. 2008; 1453-1462.

[6] Hung, CL, Chou, JCL, Ding, CM. Enhancing mobile satisfaction through integration of usability and flow. Engineering Management Research. 2012; 1(1). 44-58.

[7] Paavilainen J. Critical review on video game evaluation heuristics: social games perspective. In Proceedings of the International Academic Conference on the Future of Game Design and Technology. ACM. 2010; 56-65.

[8] Koeffel C, Hochleitner W, Leitner J, Haller M, Geven A. Tscheligi M. Using heuristics to evaluate the overall user experience of video games and advanced interaction games. Bernhaupr, R. Editor Evaluating user experience in games. London; Springer: 2010; 233-256.

[9] Browne K, Anand C. An empirical evaluation of user interfaces for a mobile video game. Entertainment Computing. 2012; 3(1):1-0(online).

[10] Soomro S, Ahmad WF, Sulaiman S. Evaluation of mobile games using playability heuristics. In Proceedings of International Visual Informatics Conference 2013 Nov.; 264-274.

[11] Oh, YB, Kim, WK. A Study on Mobile Game UI Design Element Considering User Experience -Focused on Management Simulation Game. Journal of Korean Society of Design and Culture, 2017; 23(2). 413-428.

[12] Zhao n. Kim, HH. Research on usability of mobile casual game interface - Focusing on top three casual game in Korean Google play, Journal of Digital Design, 2013; 13(4). 361-371.

[13] https://play.google.com/store/apps

[14] Korhonen H, Koivisto EM. Playability heuristics for mobile multi-player games. In Proceedings of the 2nd international conference on Digital interactive media in entertainment and arts. ACM. 2007 Sep.; 28-3.

[15] Nielsen J, Mack RL. Usability Inspection Methods. New York. John Wiley \& Sons. 1994. 
[16] Song DH, Park S, Yang SW,Yang Y, Won K. Gender Differences and Gender Stereotype in Play Style among Young Korean Gamers, Journal of the Korea Institute of Information and Communication Engineering, 2017; 21(1): 72-81.

\section{BIBLIOGRAPHY OF AUTHORS}

\begin{tabular}{|l|l|}
\hline & $\begin{array}{l}\text { Seung Hoon Lee received his B.S., M.S., and Ph. D. in Computer Science from Soongsil } \\
\text { University, Korea in 2002, 2005, 2012, respectively. He had been the president of Korea } \\
\text { Game Developer's Association during 2006-2012. He has been a professor at the } \\
\text { Department of Game Contents, Youngsan University, Korea since } 2011 \text { and he opens the } \\
\text { first ever academic Department of Virtual Reality Contents in Korea since 2017 at the } \\
\text { same university. Currently, he leads the VR contents committee in Korea Mobile Game } \\
\text { Association and related with many game exhibitions such as G-star. His research interests } \\
\text { includes software engineering, software modelling of video game design, snd VR contents } \\
\text { design. }\end{array}$ \\
\hline $\begin{array}{l}\text { Doo Heon Song received his B.S. degree in Statistics \& Computer Science from Seoul } \\
\text { National University, Korea and M.S. degree in Computer Science from the Korea } \\
\text { Advanced Institute of Science and Technology in 1983. He received his Ph.D. Certificate } \\
\text { in Computer Science from the University of California at Irvine in 1994. He has been a } \\
\text { professor at the Department of Computer Games, SongDam College, Korea, since 1997. } \\
\text { He has served as an associate editor for Journal of Multimedia Signal Processing and } \\
\text { Information Hiding and The Journal of Information and Communication Convergence } \\
\text { Engineering. His research topics include artificial intelligence, medical image processing, } \\
\text { machine learning and video game design \& culture. }\end{array}$ \\
\hline
\end{tabular}

